\title{
Misunderstanding of leptospirosis
}

\begin{abstract}
Leptospirosis is an emergent zoonosis implicating major health concern in developing countries, especially in tropical regions with frequent flooding (Watson et al., 2007). Leptospiral infections are rapidly onset and have long incubation periods of up to 28 days. Such long incubation of Leptospires leads to difficulty in identification of the medical condition as an infection-based disease, and was once misidentified as a host self-immune disorder by the clinicians. After decades of incidents, leptospirosis was first reported in 1886 as infectious disease, and Leptospira was identified as the causative bacteria in 1916 (Inada, 1908; Weil, 1886). Much confusion and misunderstanding surround the diagnosis and prevention of leptospirosis have contributed to the delayed treatment and poor prognosis.
\end{abstract}

